\title{
EDITORIAL: INTEGRATIVE SCIENCE OF HUMAN HISTORY: HOW CAN PSYCHOLOGY, ARCHEOLOGY, ANTHROPOLOGY AND BIOLOGY WORK TOGETHER
}

\author{
Jun SAIKI ${ }^{1)}$ and Atsushi IRIKI ${ }^{2)}$ \\ ${ }^{1)}$ Kyoto University, Japan \\ ${ }^{2)}$ RIKEN Center for Biosystems Dynamics Research, Japan
}

Lately launched research project of "Integrative human historical science of Out-ofEurasia: Exploring the mechanisms of the development of civilization" (Grant-in-Aid for Scientific Research on Innovative Areas; 2019-2023 [http://out-of-eurasia.jp/en/ index.html], hereafter the project) is trying to generate thorough collaborations among various academic fields of humanities and natural sciences, including archeology, anthropology, psychology, philosophy, neuroscience, biology, genomics, among potential others. Through this integrative approach, its expectation is to understand the dynamical process of the creation of human civilizations over the history, in which human as a biological organism (comprised of genes, body, brain...) produced culture, while the man-made environment and social norms formed thereby became the uniquely human niche (environment of adaptation), and thereafter acclimation to the latter produced additional changes in the former human body and cognition resulting in further modification of environment... Therefore, archaeological, historical and modern civilizations created by humans should strongly reflect the cognitive traits that evolved specific to Homo sapiens at the times. This special issue is a collection of articles to illustrate challenges of the project seeking how Psychology, Archeology, Anthropology and Biology, and other related disciplines, can work together to understand the scientific mechanisms of human history.

The first three articles are contributions from psychology to integrative human historical science. Ueda et al. (2021) propose a three-level approach to understand cultural variability and evolution of human attention, combining hypothesis-driven experiments, large-scale database, and corpus-based analyses. They hypothesize that human attentional mechanism co-evolves with objects in our environment, especially various artifacts. This article reports the initial application of the three-level approach to the study of visual search and Stroop effect. Kawabata et al. (2021) explore a novel way of collaboration between cognitive psychology and archeology, which investigated perception of archeological clay figure faces by modern humans. They discovered that perceived expression differed significantly across figures' historical periods, providing a

Correspondence concerning this article should be addressed to Jun Saiki and Atsushi Ikiri. Jun Saiki, Graduate School of Human and Environmental Studies, Kyoto University, Yoshida Nihonmatsu-cho, Sakyoku, Kyoto 606-8501, Japan (e-mail: saiki.jun.8e@kyoto-u.ac.jp); Atsushi Iriki, Laboratory for Symbolic Cognitive Development, RIKEN Center for Biosystems Dynamics Research, 6-7-3 Minatojima-minamimachi, Chuo-ku, Kobe, Hyogo 650-0047, Japan (e-mail: atsushi.iriki@riken.jp). 
basis to understand the relationship between physical properties and their psychological effects of archeological artifacts. Ishii et al. (2021) address the issue of gene-culture interaction by a replication study on interaction of social orientation and dopamine D4 receptor gene polymorphism. They failed to replicate the previous study and discussed possible ways to improve methodology to investigate gene-culture interaction. Since genetic analysis is an important tool for human historical science, development of reproducible methodology is crucial.

The next article by Iriki et al. (2021) is a nexus of psychological articles and the other articles from different disciplines such as primatology, cultural anthropology, and archeology. This paper fist introduces mutual interaction among neural, cognitive, and ecological niches in a positive feedback loop (triadic niche construction; TNC) which comprise the conceptual pillar of the project, and then advances arguments, using a multidisciplinary approach involving psychology, neurobiology, and phenomenology, to propose a shift in the mechanisms underlying TNC, from TNC-1 in hominids to TNC-2 in Homo sapiens. That is, in TNC-1 latent cognitive capabilities were incubated within its neural framework to be expressed with a simple rewiring among brain areas in TNC2, a quick and inexpensive process, that eventually result in current Anthropocene.

The following two articles discussed human history from primate research. Yamamoto (2021) explored Human uniqueness and its evolutionary basis through a comparison between humans and their closest evolutionary relatives. Examples in chimpanzees were introduced to actually have a cognitive basis for some social or cultural behaviors yet do not exhibit these in their everyday lives, which urges us to consider not only cognitive restrictions but also motivational restrictions need to be considered. Saito (2021) discusses roles of representational art in human evolution and how cognitive psychology can contribute the archeology of art. Based on her previous study of comparison of drawing behavior between chimpanzees and human children, she argues that unique characteristic of human drawing is its role as a representational schema for cultural transmission.

The last two articles are from cultural anthropology and archeology, core of human historical science. Ōnishi (2021) illustrates an ethnographic study that covers the practice of pre/non-modern craftsmanship in folk society, comprehending the mechanism of knowledge and skills which are difficult to visualize and verbalize. Such knowledge and skills are not necessarily limited to non/pre-modern societies, but shown to exist as well in contemporary societies after the Industrial Revolution. And finally, Matsumoto (2021) as a leader of the project, overviews and summarizes the history and current status of archaeological research on the mind and discusses how it leads to an integrative human historical science development, through which emphasizes an importance to focus on the human body and behavior not falling into either of biological determinism or extreme cultural relativism.

The endeavor of this special issue has tried to illustrate that, psychology and cognitive science can be expected as central for synthesizing related disciplines throughout the scope of integrative science of human history of the project. On the other hand, psychology and cognitive science, well established to the date, have commonly 
focused upon understandings of presently living humans' minds and behaviors, leaving evolutionary processes of how modern human's mind has developed over the history to form current traits relatively unexplored. Therefore, opening such historical perspectives, through collaborations with related disciplines, should contribute to enrich depth and breadth of psychology and cognitive science for their further developments towards the future generations. This special issue may represent a milestone for such future development and expansion of the research field for scientific understandings of the Human History.

\section{REFERENCES}

Iriki, A., Suzuki, H., Tanaka, S., Bretas Vieira, R., \& Yamazaki, Y. (2021). The sapient paradox and the great journey: Insights from cognitive psychology, neurobiology, and phenomenology. Psyhchologia, 63(2), 151-173. https://doi.org/10.2117/psysoc.2021-B017

Ishii, K., Masuda, T., Matsunaga, M., Noguchi, Y., Yamasue, H., \& Ohtsubo, Y. (2021). A reexamination of the effects of culture and dopamine D4 receptor gene interaction on social orientation. Psyhchologia, 63(2), 137-150. https://doi.org/10.2117/psysoc.2021-B014

Kawabata, H., Shiba, R., Matsumoto, N., Matsugi, T., \& Janik, L. (2021). How modern humans see ancient figure faces: The differential impressions and perceived expressions from clay figure faces from prehistoric and protohistoric Japan. Psyhchologia, 63(2), 116-136. https://doi.org/10.2117/psysoc. 2021-B019

Matsumoto, N. (2021). Toward an integrative human historical science of the mind, body and material. Psyhchologia, 63(2), 216-224. https://doi.org/10.2117/psysoc.2021-B021

Ōnishi, H. (2021). Dividing knowledge and skills in human history: A perspective from an ethnographic study on the folk society. Psyhchologia, 63(2), 204-215. https://doi.org/10.2117/psysoc.2021-B016

Saito, A. (2021). Archaeology of the artistic mind: From evolutionary and developmental perspectives. Psyhchologia, 63(2), 191-203. https://doi.org/10.2117/psysoc.2021-B018

Ueda, Y., Otsuka, S., \& Saiki, J. (2021). A three-level approach to understand cultural variability and the evolution of human attention. Psyhchologia, 63(2), 96-115. https://doi.org/10.2117/psysoc.2021B015

Yamamoto, S. (2021). "Unwilling” versus “unable”: Understanding chimpanzees' restrictions in cognition and motivation. Psyhchologia, 63(2), 174-190. https://doi.org/10.2117/psysoc.2021-B020 